\title{
Study of the functional mechanisms of osteopontin and chemokine-like factor 1 in the development and progression of abdominal aortic aneurysms in rats
}

\author{
JUN LI ${ }^{1,2}$, XIA BAO ${ }^{2}$, YONGXIN LI ${ }^{2}$, YUEWEI WANG ${ }^{2}$, ZONGGANG ZHAO $^{2}$ and XING JIN ${ }^{1}$ \\ ${ }^{1}$ Department of Vascular Surgery, Shandong Provincial Hospital, Shandong University, Jinan, Shandong 250021; \\ ${ }^{2}$ Department of Vascular Surgery, The Affiliated Hospital of Qingdao University, Qingdao, Shandong 266003, P.R. China
}

Received October 3, 2016; Accepted November 4, 2016

DOI: $10.3892 /$ etm.2016.3891

\begin{abstract}
The aim of the study was to investigate the functional mechanisms of osteopontin (Opn) and chemokine-like factor 1 (Cklf1) during the development and progression of abdominal aortic aneurysms (AAA) in rats. Healthy adult Sprague-Dawley rats $(n=30)$ were randomly divided into the AAA, control and sham groups (10 rats/group) and experimental rat models of AAA were generated by enzyme perfusion in abdominal aorta for $30 \mathrm{~min}$. The AAA formation was assessed by measuring the aortal diameter and hematoxylin and eosin staining as well as specific staining to detect the structural changes of the aorta and inflammatory cell infiltration. Immunohistochemistry, western blot analysis and statistical analysis were also performed to examine the expression levels of Opn, Cklf1 and matrix metalloproteinase (MMP)-2 in the arterial tissue. Rat models of AAA were successfully established by protease perfusion. After perfusion, the diameter expansion rate of abdominal aorta was significantly higher $(\mathrm{P}<0.01)$ compared to controls, elastin present at the middle layer was significantly reduced and inflammatory cell infiltration was significantly higher in AAA rats. The expression of Opn, Cklf1 and MMP-2 in the AAA group was significantly increased compared to the control group $(\mathrm{P}<0.05)$ as revealed by immunohistochemical staining. The western blot analysis revealed that, the expression levels of Opn, Cklf1 and MMP-2 in the AAA group were significantly higher than the sham and control groups $(\mathrm{P}<0.01)$. We also found that the expression of Opn and MMP-2 was positively correlated. In conclusion, in rat models of AAA, Opn and Cklf1 function synergistically to upregulate the expression of MMP-2, causing accelerated
\end{abstract}

Correspondence to: Dr Xing Jin, Department of Vascular Surgery, Shandong Provincial Hospital, Shandong University, 324 Jingwuweiqi Road, Jinan, Shandong 250021, P.R. China E-mail: jin_xing_1212@163.com

Key words: abdominal aortic aneurysm, osteopontin, chemokine-like factor 1 degradation of extracellular matrix and eventually leading to the development and progression of AAA.

\section{Introduction}

Abdominal aortic aneurysms (AAA) is a complex disorder, caused by the interaction of environmental, genetic, and biochemical factors. A variety of AAA risk factors can all lead to damage of the aortic wall, activation of inflammatory response, local inflammatory cell infiltration, degradation of extracellular matrix and massive induction of apoptosis in vascular smooth muscle cells, resulting in severe damage to the wall of the blood vessel, significant reduction of the main artery elasticity and ability to withstand pressure, and eventually the gradual swelling of the aortic wall due to failure to withstand the pressure of blood flow (1-3). Osteopontin (Opn) is a secreted glycosylated phosphoprotein, having important function in the extracellular matrix. Previous studies suggested that Opn can upregulate the expression of matrix metalloproteinases (MMPs) and thus accelerate the degradation of extracellular matrix, promoting aneurysm rupture and tumor metastasis $(4,5)$.

Chemokine-like factor 1 (Cklf1) is a newly identified cytokine by suppression subtractive hybridization method, sharing structural and functional similarity with chemokines. It has been shown that the expression of chemokines increased significantly in AAA lesions, regulating leukocyte migration towards the inflamed tissues, and promoting inflammatory reactions during the AAA progression (6,7). Cklf1 exhibited similar effects on leukocytes.

In this study, we aimed to explore the mechanisms and functions of Opn and Cklf1 in AAA in rats, based on previous findings and to provide a theoretical basis for identifying novel therapeutic targets for AAA.

\section{Materials and methods}

Materials. Porcine pancreatic elastase (E1250) was purchased from Sigma (St. Louis, MO, USA); mouse anti-rat Opn antibody was purchased from Santa Cruz Biotechnology, Inc. (Santa Cruz, CA, USA); rabbit anti-rat MMP-2 antibody was purchased from Abcam (Cambridge, UK); rabbit rCklf1 
polyclonal antibody was provided by the Department of Medicine at Peking University (Beijing, China). A total of 30 healthy male Sprague-Dawley (SD) rats weighing 150-180 g were given free access to water and standard rat chow. RNA extraction reagent TRIzol was purchased from Tiangen Biotech (Beijing) Co., Ltd. (Beijing, China), and M-MLV reverse transcriptase, TaqDNA polymerase, dNTP, primer Oligo (dT) were purchased from Takara Bio (Dalian, China). All the synthesized primers used in this study were obtained from Kang Century Biotechnology Co., Ltd. (Beijing, China). Other reagents were all of analytical grade or imported. Rats and standard rat chow were provided by the Model Animal Center of Nanjing University (Nanjing, China).

Animal groups. Thirty male SD rats, weighing $150-180 \mathrm{~g}$, were randomly divided into three groups: $\mathrm{A}, \mathrm{B}$, and $\mathrm{C} 3$ (10 rats/group). Group A was the elastase perfusion group (AAA group), perfusion with $1 \mathrm{ml}$ elastase at $20 \mathrm{U} / \mathrm{ml}$; group B was the normal saline perfusion group (control group), and group $\mathrm{C}$ was the untreated group (sham group). The surgical procedure included the following steps: Anesthesia, laparotomy, isolation of abdominal aorta within $0.5-1.0 \mathrm{~cm}$, intubation through upper abdominal aortic bifurcation mouth, perfusion by high pressure, perfusion liquid varies due to experimental groups, and the abdomen was repaired at the end of the surgery.

Pathological stain. Specimens were fixed with $4 \%$ paraformaldehyde and stained with hematoxylin and eosin (H\&E) and Victoria blue to label elastic fibers in blue-green and collagen fibers in yellow.

Immunohistochemistry. Conventional avidin-biotin complex method was used to detect the distribution of Opn in different AAA layers with mouse anti-rat Opn antibody (Abcam, Cambridge, MA, USA; dilution 1:500; cat. no.: ab69498) and goat anti-mouse secondary antibody (Abcam; dilution 1:500; cat. no.: ab6789).

Western blotting. Tissue samples stored in liquid nitrogen at $-80^{\circ} \mathrm{C}$ were homogenized followed by protein extraction and denaturation. Protein concentration was determined using a Coomassie brilliant blue colorimetric assay and proteins separated by sodium dodecyl sulphate-polyacrylamide gel electrophoresis (SDS-PAGE). After completion of the electrophoresis and rinsing gel with deionized water, the separated proteins were transferred to nitrocellulose membrane and blocked in a $5 \%$ non-fat milk powder at $4^{\circ} \mathrm{C}$ overnight. Subsequently, the blocking solution was removed and the membrane was rinsed with TBST 3 times. Primary antibody (dilution of 1:500) was then incubated with the membrane at room temperature on a shaker for $2 \mathrm{~h}$ followed by rinsing with TBST, and incubation with horseradish peroxidase-labeled goat anti-mouse secondary antibody on a shaker at room temperature for $1 \mathrm{~h}$. The diaminobenzidine (DAB) chromogenic detection system was used for signal visualization and pro 4.0 gel absorbance measurement/analysis software was used to perform protein quantification.

Results evaluation. Sections treated with specific staining and immunohistochemical staining without hematoxylin
Table I. Diameter changes in abdominal aorta $(\mathrm{mm})$.

\begin{tabular}{lrccrl}
\hline Groups & No. & Before & After & T-value & P-value \\
\hline AAA & 9 & $5.23 \pm 1.28$ & $12.42 \pm 6.81^{\mathrm{a}, \mathrm{b}}$ & 12.87 & 0.008 \\
Control & 10 & $5.29 \pm 1.29$ & $5.92 \pm 1.21$ & 10.98 & 0.048 \\
Sham & 10 & $5.33 \pm 2.18$ & $5.23 \pm 3.23$ & 0.69 & 0.32 \\
\hline
\end{tabular}


${ }^{\mathrm{b}}$ significantly different compared to the sham group, $\mathrm{P}<0.01$. AAA, abdominal aortic aneurysms.

were analyzed using a microscope (Olympus, Tokyo, Japan) at a magnification of x100. Ten visual fields were randomly selected, followed by analysis using a computer image system. Detection criteria was the average absorbance value of positive staining multiplied by relative area of staining (s \%).

Statistical analysis. SPSS 13.0 statistical software (SPSS, Inc., Chicago, IL, USA) was used for data analysis. Results were presented as mean \pm standard deviation. The t-test and variance analysis was performed to assess significant difference. The ANOVA single factor analysis of variance was used for comparing multiple groups of data. A correlation analysis was performed using Spearman correlation analysis.

\section{Results}

Abdominal aortic diameter change. All the rats survived except one rat from the AAA group, which died after surgery. On the 14th day after surgery, abdominal aortic dilatation in rats from the AAA group was 2.38-fold its original aortic diameter, while in rats from the control group, abdominal aortic dilatation was only 1.12-fold the original and there was no abdominal aortic dilatation in rats from the sham group. The t-test analysis revealed that compared to the control group (group B) and sham group (group C), the abdominal aortic dilatation ratio in rats from the AAA group (group A) was significantly higher $(\mathrm{P}<0.01)$, while there was no significant difference in the dilatation ratio between groups $\mathrm{B}$ and $\mathrm{C}$ $(\mathrm{P}>0.05$; Table I). Ultrasonography is shown in Fig. 1.

Staining. H\&E staining showed that the arterial wall in rats in the AAA group was thickened, was damaged and had a broken structure. Debris and notch and the smooth muscle cells were disorganized with massive inflammatory cell infiltration. On the other hand, the arterial wall from the control and sham groups looked much better in terms of thickness, structural integrity, continuous resistance, smooth muscle cell distribution and the number of inflammatory cells (Fig. 2A-C). Elastic fibers staining showed that in the AAA group, elastic fibers in artery medial layer were badly damaged with irregular morphology, disordered and reduced layers, loss of normal curvature and continuity, appearance of fracture, fragmentation and notch, whereas in the control and sham group, elastic fibers remained relatively intact, resembling regular waves with distinct layers, fine continuity and curvature (Fig. 2D-F). Opn-specific protein staining was strongly positive within aneurysms vascular structure in the 


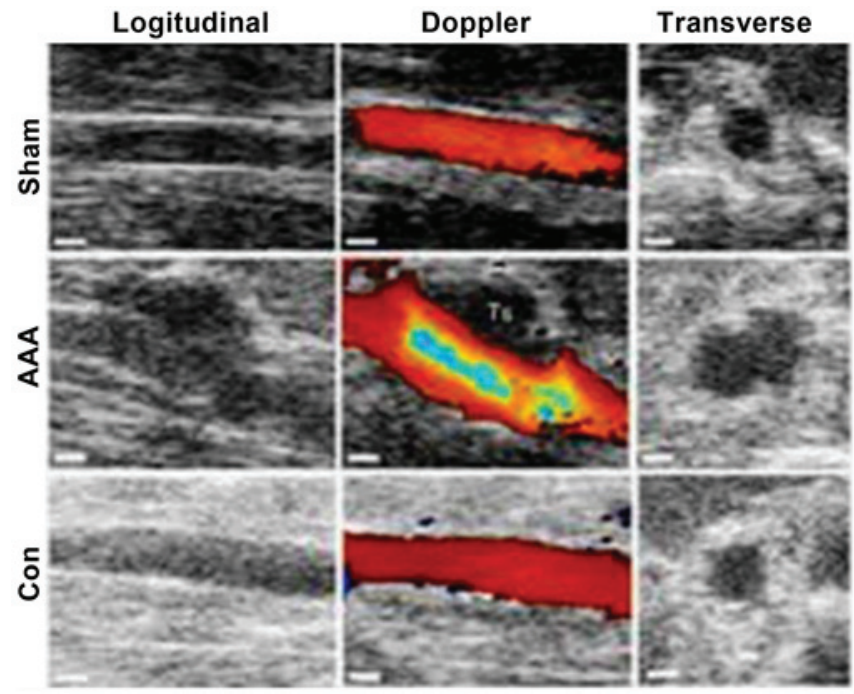

Figure 1. Ultrasonography of abdominal aorta shows that the transverse diameter of the aorta in the abdominal aortic aneurysms (AAA) group significantly increased on day 14 after surgery, and the directions of blood flow were not consistently monitored by Doppler ultrasound. By contrast, the transverse diameter of the aorta was normal in the sham and control (Con) groups and only one-way blood flow was evident on the Doppler ultrasonography.
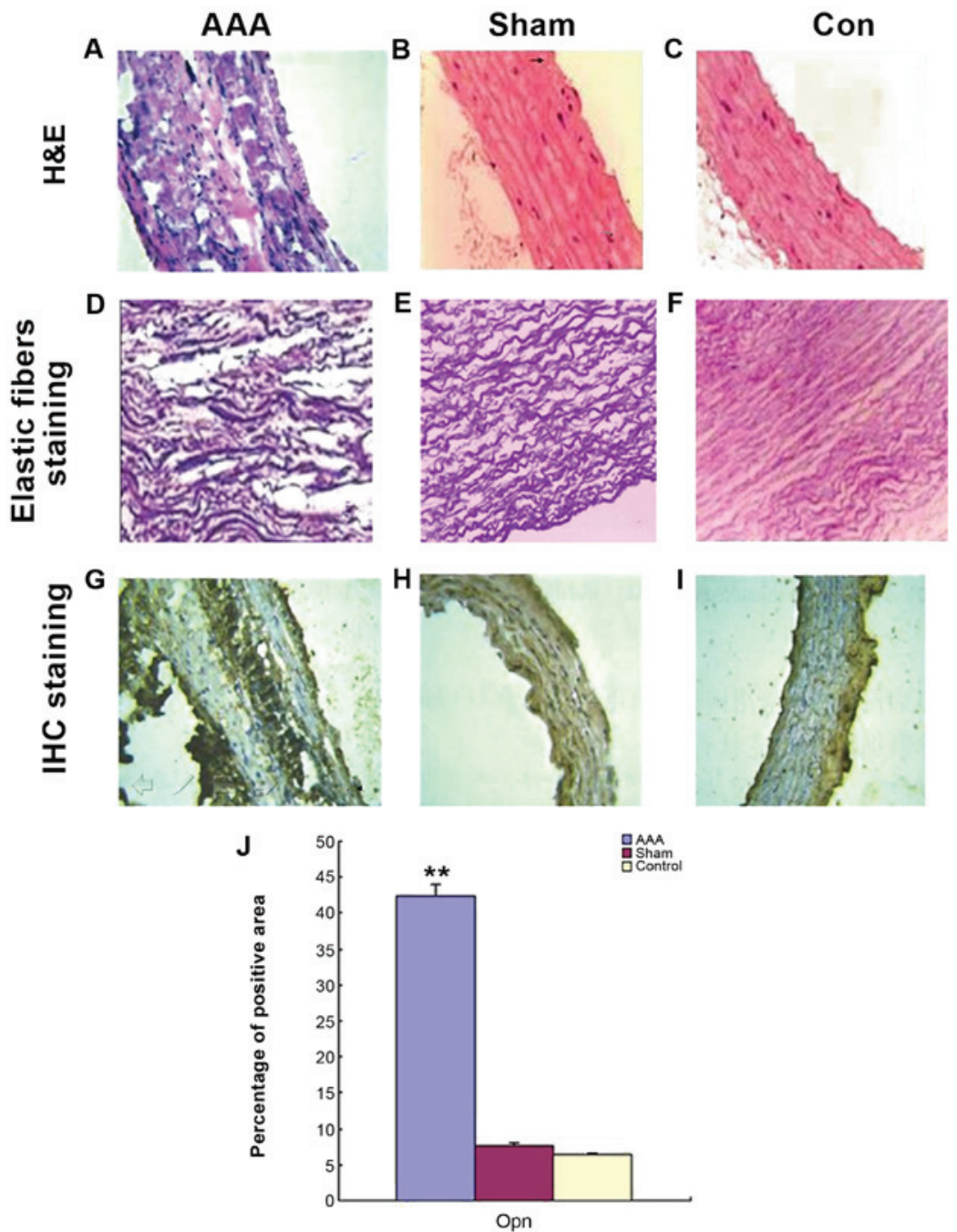

Figure 2. Hematoxylin and eosin (H\&E) staining of the arterial wall. (A) Thickening of the arterial wall with disorganized smooth muscle cells and massive inflammatory cell infiltration in the abdominal aortic aneurysms (AAA) group. (B and C) Normal thickness and structural integrity of the arterial wall in the control (Con) and normal group.s (D) Elastic fibers staining in the AAA group demonstrating irregular morphology of badly damaged elastic fibers in artery media. (E and F) Relatively intact elastic fibers in the sham and control groups. (G) Strong Opn-specific protein staining within aneurysm vascular structure in the AAA group, and (H and I) weak Opn expression in the sham and control groups. (J) Immunohistochemical staining in the AAA group. Opn expression levels in rats in the AAA group markedly increased compared with the control and sham groups and the difference was statistically significant $\left({ }^{* *} \mathrm{P}<0.05\right)$. 
AAA group with a high expression located at the medial aneurysm wall (serious disease area with fracture, fragment and notch) (Fig. 1C and D). By contrast, in the control and sham group, Opn expression was weak. Statistical analysis is shown in Fig. 2E and Table II.

Western blotting. Expression of Opn, rCklf1 and MMP-2 was elevated in the AAA group, while in the control and sham groups, the expression of all three proteins was low. Analysis revealed that the expression of Opn, rCklf1 and MMP-2 was higher in the AAA group than that in the control and sham group and the difference was statistically significant $(\mathrm{P}<0.05)$. However, the difference was not considered significant between the control and sham group ( $\mathrm{P}>0.05$; Table II). Additionally, the expression of Opn, rCklf1 and MMP-2 was positively correlated with each other (Table III).

\section{Discussion}

Aortic aneurysm primarily affects male populations over the age of 55 with a mortality rate as high as $50-80 \%$ if a rupture occurs, making it clinically significant to investigate the mechanism controlling mthe initiation and progression of AAA as well as to improve clinical outcomes in patients (8). At present, scholars at home and abroad have created AAA rat models through a range of methods including genetic defect induction, calcium chloride-mediated injury and elastase perfusion (9). Among these methods, the elastase perfusion model is superior in mimicking the native pathological conditions in AAA patients (10). In the present study, the AAA rat model was successfully generated using a micro-surgery method. Victoria blue staining revealed the disappearance of elastic fibers, degeneration of vascular muscle cells and a significant increase in collagen fibers, in agreement with human AAA pathological conditions. Previous findings have shown that inflammation plays an important role in the pathogenesis of AAA and the regulation of monocyte-macrophage cells, lymphocyte activation and migration by chemokine is one of the key steps to develop AAA $(11,12)$. Additionally, the expression of chemokines was significantly increased in AAA patients and macrophages as well as lymphocytes in blood infiltrating the arterial wall when induced by chemokines, causing the release of excess MMP and further degradation of structural proteins (elastin, collagen) in the aorta wall and damage of elastic fibers and collagen fibers (13). Cklf, a newly identified chemokine, has exhibited chemotactic activities on neutrophils, lymphocytes and monocyte in vitro and in vivo. In addition, Cklf is involved in the regulation of endometrial hyperplasia, atherosclerosis and other inflammatory processes $(14,15)$.

In the present study, the rat AAA models have been successfully created by elastase perfusion surgery. In AAA rats, the aortic diameter expansion rate after surgery was significantly higher $(\mathrm{P}<0.01)$, elastin in medial layer was significantly reduced and inflammatory cell infiltration was increased significantly compared to the control rats. In addition, immunohistochemical staining showed that the expression of Opn, Cklf1 and MMP-2 in the AAA group was significantly increased $(\mathrm{P}<0.05)$. Furthermore, western
Table II. Expression of Opn, rCklf1 and MMP-2 examined by western blotting.

\begin{tabular}{lccc}
\hline Group & Opn, x10 & rCklf1, x10 & MMP-2, x 10 \\
\hline AAA & $2.78 \pm 2.81$ & $6.02 \pm 6.78$ & $9.37 \pm 6.72$ \\
Sham & $4.57 \pm 1.27$ & $4.78 \pm 4.17$ & $8.98 \pm 7.28$ \\
Control & $2.18 \pm 1.94$ & $4.37 \pm 7.02$ & $2.76 \pm 3.47$ \\
\hline
\end{tabular}

Opn, osteopontin; rCklf1, rat chemokine-like factor 1; MMP, matrix metalloproteinase; AAA, abdominal aortic aneurysms.

Table III. Spearman correlation analysis.

\begin{tabular}{|c|c|c|c|c|c|c|}
\hline \multirow[b]{3}{*}{ Group } & \multicolumn{6}{|c|}{ Western blotting } \\
\hline & \multicolumn{2}{|c|}{ Opn } & \multicolumn{2}{|c|}{ rCklf1 } & \multicolumn{2}{|c|}{ MMP-2 } \\
\hline & P-value & r-value & P-value & r-value & P-value & r-value \\
\hline Opn & - & - & $<0.01$ & 0.885 & $<0.05$ & 1.298 \\
\hline rCklf1 & $<0.01$ & 0.876 & - & - & $<0.01$ & 1.045 \\
\hline MMP-2 & $<0.01$ & 0.576 & $<0.01$ & 0.028 & - & - \\
\hline
\end{tabular}

Opn, osteopontin; rCklf1, rat chemokine-like factor 1; MMP, matrix metalloproteinase.

blot analysis revealed that the expression of Opn, Cklf1 and MMP-2 in the AAA group was significantly higher than that in the sham and control groups $(\mathrm{P}<0.01)$ and $\mathrm{Opn}$ as well as rCklf1 was positively correlated with MMP-2; rs (Opn)=1.298, rs $(\mathrm{MMP}-2)=1.045(\mathrm{P}<0.05)$. The results of the present study suggest that Opn, and rCklf1 upregulate the expression of extracellular matrix MMP and elevated MMP destroys the balance between anabolic and catabolic activity in extracellular matrix outside the arterial wall. Thus, the degradation of extracellular matrix is accelerated and the pathogenesis of AAA is promoted. Previous studies suggested that the occurrence of aortic aneurysms involved the decomposition and denaturation of arterial elastin, collagen and other factors, resulting in damage in arterial medial layer and the support plate (16). Under the impact of high blood flow, the release of MMP and other proteinases from macrophages and aortic smooth muscle cells were further accelerated. Additionally, widely infiltrated lymphocytes and macrophages in the aortic wall produced a variety of cytokines (such as interleukins, tumor necrosis factor and immunoreactive fibronectin) (17) as well as immunoglobulins, which in turn lead to activation of the protease cascade. Opn levels increased at the inflammatory lesion in chronic inflammatory and autoimmune diseases caused by injury, and this upregulation was more evident in particular in activated $\mathrm{T}$ lymphocytes, mononuclear macrophages and adjacent parts (18).

In summary, findings of the present study have shown that, in the rat model of AAA, Opn and Cklf1 synergistically upregulated the expression of MMP-2 in tumor tissue, which 
in turn, accelerated the degradation of extracellular matrix, eventually resulting in the development of aortic aneurysms.

\section{References}

1. Chen HZ, Wang F, Gao P, Pei JF, Liu Y, Xu TT, Tang X, Fu WY, $\mathrm{Lu} \mathrm{J}$, Yan YF, et al: Age-associated sirtuin 1 reduction in vascular smooth muscle links vascular senescence and inflammation to abdominal aortic aneurysm. Circ Res 119: 1076-1088, 2016.

2. Schaheen B, Downs EA, Serbulea V, Almenara CC, Spinosa M Su G, Zhao Y, Srikakulapu P, Butts C, McNamara CA, et al: B-Cell Depletion Promotes Aortic Infiltration of Immunosuppressive Cells and Is Protective of Experimental Aortic Aneurysm. Arterioscler Thromb Vasc Biol 36: 2191-2202, 2016.

3. Shingaki M, Kato M, Motoki M, Kubo Y, Isaji T and Okubo N: Endovascular repair for abdominal aortic aneurysm followed by type B dissection. Asian Cardiovasc Thorac Ann: Sep 15, 2016.

4. Tan Y, Wang Y, Li L, Xia J, Peng S and He Y: Chemokine-like factor 1-derived $\mathrm{C}$-terminal peptides induce the proliferation of dermal microvascular endothelial cells in psoriasis. PLoS One 10: e0125073, 2015.

5. Moores AE, Cahill MS and Villines TC: Myocardial infarction and aortic root mycotic aneurysm complicating aortic valve endocarditis: Utility of cardiac CT. Case Rep Med 2016: 3756302 , 2016.

6. Ijaz T, Tilton RG and Brasier AR: Cytokine amplification and macrophage effector functions in aortic inflammation and abdominal aortic aneurysm formation. $\mathbf{J}$ Thorac Dis 8: E746-E754, 2016.

7. Gomez I, Ozen G, Deschildre C, Amgoud Y, Boubaya L, Gorenne I, Benyahia C, Roger T, Lesèche G, Galardon E, et al: Reverse regulatory pathway (H2S/PGE2/MMP) in human aortic aneurysm and saphenous vein varicosity. PLoS One 11: e0158421, 2016.

8. Yuan SM, Wang J, Huang HR and Jing H: Osteopontin expression and its possible functions in the aortic disorders and coronary artery disease. Rev Bras Cir Cardiovasc 26: 173-182, 2011.
9. Tu M,Li Y,Zeng C, Deng Z, Gao S, Xiao W, Luo W, Jiang W, Li L and Lei G: MicroRNA-127-5p regulates osteopontin expression and osteopontin-mediated proliferation of human chondrocytes. Sci Rep 6: 25032, 2016.

10. Shang T, Ran F, Qiao Q, Liu Z and Liu CJ: Tanshinone IIA attenuates elastase-induced AAA in rats via inhibition of MyD88-dependent TLR-4 signaling. Vasa 43: 39-46, 2014.

11. Tarín C, Fernández-Laso V, Sastre C, Madrigal-Matute J, Gómez M, Zaragoza C, Egido J, Burkly LC, Martín-Ventura JL and Blanco-Colio LM: Tumor necrosis factor-like weak inducer of apoptosis or Fn14 deficiency reduce elastase perfusion-induced aortic abdominal aneurysm in mice. J Am Heart Assoc 3: e000723, 2014

12. Yang GY, Chen X, Sun YC, Ma CL and Qian G: Chemokine-like factor 1 (CLFK1) is over-expressed in patients with atopic dermatitis. Int J Biol Sci 9: 759-765, 2013.

13. Thompson RW and Baxter BT: MMP inhibition in abdominal aortic aneurysms. Rationale for a prospective randomized clinical trial. Ann N Y Acad Sci 878: 159-178, 1999.

14. Wang ZZ, Zhang Y, Yuan YH and Chen NH: Developmental expression of chemokine-like factor 1 , a novel member of chemokines family, in postnatal rat cerebral cortex. Neurosci Lett 519: 51-55, 2012.

15. Zheng Y, Guo C, Zhang Y, Qi H, Sun Q, Xu E, Zhang Y, Ma D and Wang Y: Alleviation of murine allergic rhinitis by $\mathrm{C} 19$, a C-terminal peptide of chemokine-like factor 1 (CKLF1). Int Immunopharmacol 11: 2188-2193, 2011.

16. Carmo M, Colombo L, Bruno A, Corsi FR, Roncoroni L, Cuttin MS, Radice F, Mussini E and Settembrini PG: Alteration of elastin, collagen and their cross-links in abdominal aortic aneurysms. Eur J Vasc Endovasc Surg 23: 543-549, $2002 \S$.

17. Martín-Alonso M, García-Redondo AB, Guo D, Camafeita E, Martínez F, Alfranca A, Méndez-Barbero N, Pollán Á, Sánchez-Camacho C, Denhardt DT, et al: Deficiency of MMP17/MT4-MMP proteolytic activity predisposes to aortic aneurysm in mice. Circ Res 117: e13-e26, 2015.

18. Wang C, Chang Q, Qian X, Tian C and Sun X: Angiotensin II induces an increase in MMP-2 expression in idiopathic ascending aortic aneurysm via AT1 receptor and JNK pathway. Acta Biochim Biophys Sin (Shanghai) 47: 539-547, 2015. 